\title{
Uptake of carrier testing in families after cystic fibrosis diagnosis through newborn screening
}

\author{
Belinda J McClaren ${ }^{1,2,3}$, Sylvia A Metcalfe ${ }^{\star, 1,2}$, MaryAnne Aitken ${ }^{2,4}$, R John Massie ${ }^{2,5,6}$, \\ Obioha C Ukoumunne ${ }^{2,7}$ and David J Amor ${ }^{2,3,8}$
}

\begin{abstract}
Newborn screening (NBS) for cystic fibrosis (CF) provides the opportunity for cascade carrier testing of relatives. Uptake of testing by adult non-parent relatives of children diagnosed with CF through NBS has not been previously described, and this study describes uptake by both parents and adult non-parent relatives in Victoria, Australia. Pedigrees were taken from parents of children who were born in 2000-2004 and diagnosed with CF. A total of 40 families were eligible for the study and 30 (75\%) were recruited. In all, 716 non-parent relatives were identified from the pedigrees as eligible for carrier testing, and 82 (adjusted uptake percentage: $11.8 \%$; 95\% confidence interval 8.0-15.7) have had carrier testing by March 2009. On average, 2.7 non-parent relatives per family had CF carrier testing after diagnosis through NBS. The odds of being tested were greater for females than males (adjusted odds ratio $1.61 ; 95 \%$ confidence interval $1.11-2.33 ; P=0.01$ ) and greater for those more closely related to the child with CF (adjusted odds ratio $5.17 ; 95 \%$ confidence interval $2.38-11.24 ; P<0.001$ ). Most relatives who undergo testing are tested immediately after the baby's diagnosis; however, some testing is undertaken up to 8 years later. These results indicate that in a clinical setting, the diagnosis of a baby with CF by NBS does not lead to carrier testing for the majority of the baby's non-parent relatives. We suggest re-contact with parents to offer cascade carrier testing.
\end{abstract}

European Journal of Human Genetics (2010) 18, 1084-1089; doi:10.1038/ejhg.2010.78; published online 26 May 2010

Keywords: cystic fibrosis; carrier testing; newborn screening; genetic testing; CFTR

\section{INTRODUCTION}

Cystic fibrosis (CF) is the most common severe recessive condition in children, with a birth prevalence of approximately 1 in 3500 for Caucasian populations. ${ }^{1} \mathrm{CF}$ is characterized by suppurative lung disease, pancreatic exocrine insufficiency and elevated sweat electrolytes. ${ }^{2}$ Advances in treatment have seen life expectancy increase to approximately 30 years of age, but as yet there is no cure for CF. The $\mathrm{CF}$ carrier frequency is approximately 1 in 25 in Caucasian populations; however, those with a family history of CF are at greater risk. The identification of the Cystic fibrosis transmembrane conductance regulator (CFTR) gene in 1989 made possible the detection of $\mathrm{CF}$ carriers. ${ }^{3}$ The subsequent inclusion of CFTR mutation analysis in newborn screening (NBS) programs allowed genetic diagnosis and the availability of cascade carrier testing for family members. Knowledge of carrier status provides information that may be used to make reproductive decisions. Prenatal diagnosis is available for carrier couples, who may choose to terminate an affected pregnancy, or continue with the pregnancy and use the information to prepare for the birth of their child with CF. Knowledge of carrier status before pregnancy means there are some other options available for carrier couples, including preimplantation genetic diagnosis.

It has been recommended that carrier testing be made available for adults with a family history of $\mathrm{CF}^{4,5}$ Several studies have explored issues associated with offering carrier testing to people who have a family history of CF. ${ }^{6-10}$ These studies that have been undertaken in research settings involved intervention from the investigators, such as directly contacting relatives, or giving patients resources to communicate with family members after a genetic counselling appointment. The extent to which non-parent relatives access carrier testing in the context of a clinical setting, rather than in response to a specific research study-driven offer of testing, has not previously been explored. One such context is carrier testing of adult non-parent relatives after a child's diagnosis of CF through NBS. Carrier testing is offered to adult relatives, including parents, of a child diagnosed with CF through NBS in Victoria, Australia. ${ }^{11,12}$ Given the implementation of CF NBS in other clinical settings worldwide, it is necessary to explore cascade carrier testing in the context of a diagnosis after NBS rather than based on family history after the clinical diagnosis of a symptomatic child. This study, in a context in which CF NBS is well established, can provide information for these other settings regarding the likely uptake of cascade carrier testing by non-parent adult relatives. The aim of this study was to describe the uptake of carrier testing, in a clinical setting, by adult non-parent relatives of children diagnosed with CF through NBS.

\section{MATERIALS AND METHODS}

Setting

In Australia, there are approximately 90 children born with CF each year. In the State of Victoria, Australia, CF has been included in the NBS program

${ }^{1}$ Genetics Education and Health Research, Murdoch Childrens Research Institute, Melbourne, Victoria, Australia; ${ }^{2}$ Department of Paediatrics, University of Melbourne, Melbourne, Victoria, Australia; ${ }^{3}$ Genetic Health Services Victoria, Murdoch Childrens Research Institute, Melbourne, Victoria, Australia; ${ }^{4}$ Research Strategy, Murdoch Childrens Research Institute, Melbourne, Victoria, Australia; ${ }^{5}$ Department of Respiratory Medicine, Royal Children's Hospital, Melbourne, Victoria, Australia; ${ }^{6}$ Respiratory Diseases, Murdoch Childrens Research Institute, Melbourne, Victoria, Australia; ${ }^{7}$ Clinical Epidemiology and Biostatistics Unit, Murdoch Childrens Research Institute, Melbourne, Victoria, Australia; ${ }^{8}$ Genetic Health Research, Murdoch Childrens Research Institute, Melbourne, Victoria, Australia

${ }^{*}$ Correspondence: Professor SA Metcalfe, Genetics Education and Health Research, Murdoch Childrens Research Institute, 10th Floor, Royal Children's Hospital, Flemington Road, Parkville, Melbourne, Australia. Tel: +61 38341 6309; Fax: +61 38341 6212; E-mail: sylvia.metcalfe@mcri.edu.au

Received 28 July 2009; revised 31 March 2010; accepted 21 April 2010; published online 26 May 2010 
since 1989. All babies are screened using a heel prick blood sample collected soon after birth. The screen identifies elevated immunoreactive trypsinogen (IRT), followed by CFTR mutation analysis and sweat testing. ${ }^{13}$ Some babies who are CF carriers will be detected; exploration of the effect of carrier detection through NBS has been previously reported. ${ }^{14-16}$ After a diagnosis of CF through NBS, parents are offered an intensive education program through the CF clinic at the Royal Children's Hospital, Melbourne. ${ }^{17}$ During this time, they have the opportunity to see a genetic counsellor who discusses the implications of the diagnosis for subsequent pregnancies and the availability of cascade carrier testing for family members. ${ }^{17} \mathrm{~A}$ family pedigree is drawn and parents are provided with a letter for distribution within the family to assist them with discussing carrier testing. In Victoria, CF carrier testing is performed by a single state-wide laboratory, the Victorian Clinical Genetics Service, and is free of charge for family members (funded by the State Department of Human Services).

\section{Ethics approval}

This study was approved by the Royal Children's Hospital Ethics Committee (HREC 27121C) and Genetic Health Services Victoria, the Victorian Clinical Genetics Service.

\section{Recruitment}

From August 2008 to February 2009, parents of children who were diagnosed with CF through NBS in Victoria, managed by the clinical service, and who were born between 1 January 2000 and 31 December 2004, were invited to participate in the study $(n=82)$. This 5 -year period was chosen because it was recent enough to anticipate that contact details of families would be current and distant enough for family members to have had carrier testing. A letter of invitation was sent with a newsletter from the CF clinic, and a member of the research team approached parents at their child's next appointment. An interview was scheduled with parents who chose to participate. Before the interview, the family's genetics file, a medical record documenting contacts with the genetics service, was accessed to obtain the pedigree that was collected at the time of the child's diagnosis. Parents were interviewed to validate the pedigree from the genetics file. Validation involved checking that all relatives were included in the pedigree, and confirming names, ages and locations of relatives.

\section{Data collection}

Information from the pedigrees was entered into a Microsoft Access database including: first name, family name, whether from the maternal or paternal side of the CF child's family, relationship to child, carrier risk (based on relationship to child), gender and date of birth. To determine whether relatives had a carrier test, the centralized Victorian Clinical Genetics Service laboratory database was searched at the end of March 2009 using the information collected for each eligible individual.

\section{Inclusion and exclusion criteria}

Eligibility criteria for inclusion of relatives were: the familial mutation was detectable; the relative was a genetic relative; and the carrier risk based on relationship to child was 1,1 in 2, 1 in 4 or 1 in 8 . Three generations of the family were included (ie, up to and including grandparental generation). Relatives were excluded if they were known to reside interstate or overseas (as their test records would not be available on the Victorian Clinical Genetics Service pathology laboratory database), if they were deceased at the time of the child's diagnosis or if they were $<18$ years of age by March 2009 .

\section{Data analysis}

All analyses were performed using Stata 10.1 (StataCorp., Stata Statistical Software: Release 10.1, Stata Corporation, College Station, TX, USA). We report uptake of CF carrier testing as the percentage of eligible relatives who have had carrier testing. Estimation of uptake has been adjusted for clustering because each individual in the data set is part of a family, and therefore cannot be considered independent. For example, it is expected that an individual who is part of a family in which several people have had carrier testing is more likely to also have carrier testing. This correlation, or clustering, between the uptake of testing of participants from the same family needs to be accounted for, otherwise confidence intervals for the estimated percentage tested will be too narrow, implying a greater degree of precision than there actually is. ${ }^{18}$ The regression-based method of marginal models using generalized estimating equations (GEE) with information sandwich (robust) estimates of standard error was used to estimate the percentage of family members who have had carrier testing with a $95 \%$ confidence interval that is adjusted for clustering. ${ }^{18,19}$ In this analysis, an exchangeable correlation structure was assumed and the 'identity' link function was selected to estimate the proportion of relatives who have had carrier testing. Unadjusted and adjusted (multivariable) logistic regression models were fitted using the GEE method to investigate the relationship between uptake of carrier testing for adult non-parent relatives and the following three variables: gender, carrier risk based on relationship to child and maternal versus paternal family status. We present median and interquartile range (IQR) for the time taken by parents and other relatives to have carrier testing, and the age at which testing occurred.

\section{RESULTS}

\section{Description of sample}

A total of 40 eligible children attended the CF clinic during the 7-month recruitment period from August 2008 to February 2009, and their parents were invited to participate. Parents of 36 children consented to take part. Of the four families who declined participation immediately, three were too busy and one did not wish to bring up these issues again in the family. Interviews were held with 30 of the 36 families (participation rate of 75\%). Of the six families who were not interviewed, four could not be reached by telephone to organize the interview (did not answer calls or return messages), one declined participation because of a change in family situation (recent separation) and one left a telephone message to say they had changed their mind about participating. Pedigrees from the 30 participating families were examined for this audit, from which 775 relatives, including parents, eligible for carrier testing were identified. A further 85 relatives resided interstate or overseas and were excluded from the study, as their testing details were not available and could not be determined. The median (IQR) family size was 29 (22-37) members, with a range from 2 to 60 members.

\section{Uptake of carrier testing}

Of the 775 eligible relatives, 120 (15.5\%) have had carrier testing. Table 1 shows the proportion undergoing testing for each group defined by carrier risk based on relationship to the child with CF. Of the 716 non-parent relatives, $82(11.5 \%)$ had carrier testing. On average, this equates to $2.7(82 / 30)$ people per family (other than

Table 1 Number of eligible relatives who had carrier testing based on their estimated carrier risk as determined by their relationship to the child with CF

\begin{tabular}{llr}
$\begin{array}{c}\text { Carrier risk based } \\
\text { on relationship to } \\
\text { the child with CF }\end{array}$ & Relative & $\begin{array}{c}\text { Number tested/total } \\
\text { eligible (\%) }\end{array}$ \\
\hline 1 & Parents & $38 / 59(64.4 \%)$ \\
1 in 2 & Grandparents & $22 / 94(23.4 \%)$ \\
& Aunts/uncles & $37 / 95(38.9 \%)$ \\
in 4 & Great aunts/uncles & $15 / 264(5.7 \%)$ \\
& First cousins & $4 / 26(15.4 \%)$ \\
& Half sibling & $1 / 2(50.0 \%)$ \\
1 in 8 & Half aunt/uncle & $1 / 13(7.7 \%)$ \\
& First cousins once removed & $2 / 221(1.0 \%)$ \\
Total & Half first cousin & $0 / 1(0.0 \%)$ \\
\hline
\end{tabular}

aChildren of great aunts/uncles. 
the parents) who have had carrier testing after diagnosis of CF through NBS. After adjusting for clustering, the estimated proportion tested was $16.3 \%$ (95\% CI 12.1-20.5) for all relatives, including parents, and $11.8 \%$ (95\% CI 8.0-15.7) for the 716 non-parent relatives. These estimates are slightly larger than the observed percentages because of the relative down-weighting of the contribution made by larger families ( $>20$ members), and the relative up-weighting of the contribution made by smaller families ( $\leq 20$ members) in the analyses that are adjusted for clustering. Because most of the larger families had lower uptake, $15.4 \%$ (95\% CI 11.0-19.8) compared with smaller families $19.6 \%$ (95\% CI $0.8-31.5)$, the clustering-adjusted estimates are larger.

\section{Description of parents of children with CF}

Of 59 parents of children with CF, 38 (64.4\%) have had carrier testing, and in 18 families both parents were tested. For 9 children who were homozygous for p.F508del, neither parent was tested (Table 2). The median (IQR) time taken for parents to be tested was 1.3 (1.1-1.6) months with a range of 0.6-43.6 months. The median (IQR) age of parents when tested was $32.1(27.1-35.0)$ years, with a range of $17.2-41.6$ years.

Predictors of uptake of carrier testing among non-parent relatives The results from the logistic regression of test status among nonparent relatives of the child with CF are shown in Table 3. Relatives with a carrier risk of 1 in 8 , based on relationship to the child with $\mathrm{CF}$, were not included in this analysis because the sample size in this group was too small. In the unadjusted analysis, the odds of having the test were 1.6 times greater for female relatives than for males. The odds of testing were over 5 times greater for family members with a carrier risk of 1 in 2 than for those with a carrier risk of 1 in 4 . There was no strong evidence of an association between being tested and maternal versus paternal family member status. In the adjusted model, the associations of gender $(P=0.01)$ and carrier risk based on relationship to the child with $\mathrm{CF}(P<0.001)$ remained.

\section{Time taken to be tested and age when tested of non-parent} relatives after CF diagnosis through NBS

The median (IQR) time taken to be tested from the child's date of birth to the date of a non-parent relative's test was 4.8 (2.8-38.2) months (Figure 1). The median (IQR) age of non-parent relatives when tested was 39.1 (29.8-56.6) years, with a range of 15.8-82.7 years, and shows a bimodal distribution (Figure 2).

\section{DISCUSSION}

This is the first study to examine the uptake of CF carrier testing by adult non-parent relatives of a child diagnosed with CF through NBS. On average, three non-parent relatives have carrier testing in the years after a child's diagnosis of CF through NBS. However, the vast majority $(88.5 \%)$ of eligible non-parent relatives remain untested. In this sample, a greater proportion of females were tested than males, consistent with a previous study on the role of gender in decision

Table 2 Genetic mutation profile of children in sample and the test status of parents

\begin{tabular}{|c|c|c|c|c|c|}
\hline Genetic mutation combination & Number of children & Both parents tested & Mother only tested & Father only tested & Neither parent tested \\
\hline p.F508del/p.F508del & 20 & 9 & $1^{a}$ & 1 & 9 \\
\hline p.F508del/c.489+1G>T & 3 & 2 & $1^{\mathrm{b}}$ & 0 & 0 \\
\hline p.F508del/p.G542X & 1 & 1 & 0 & 0 & 0 \\
\hline p.F508del/p.G551D & 2 & 2 & 0 & 0 & 0 \\
\hline p.F508del/p.N1303K & 2 & 2 & 0 & 0 & 0 \\
\hline p.F508del/unknown ${ }^{\mathrm{c}}$ & 1 & 1 & 0 & 0 & 0 \\
\hline p.1507del/unknownc & 1 & 1 & 0 & 0 & 0 \\
\hline
\end{tabular}

aNo test data for father.

${ }^{5}$ The mother was found to carry the c. $489+1 \mathrm{G}>\mathrm{T}$ mutation.

cOnly relatives of the parent for whom the mutation was detectable were included in this study of uptake of carrier testing.

Table 3 Logistic regression of test status of non-parent relatives on potential predictors of uptake of carrier testing

\begin{tabular}{|c|c|c|c|}
\hline & Proportion tested (\%) & Unadjusted analysis OR; 95\% Cl; P-value & Multivariable analysis AOR; 95\% Cl; P-value \\
\hline \multicolumn{4}{|c|}{ Gender of relative ${ }^{\mathrm{a}}$} \\
\hline Female & 14.5 & $1.64 ; 1.20-2.25 ; 0.002$ & $1.61 ; 1.11-2.33 ; 0.01$ \\
\hline Male & 9.45 & Reference group & Reference group \\
\hline \multicolumn{4}{|c|}{ Maternal or paternal relatives } \\
\hline Paternal & 12.1 & $1.19 ; 0.66-2.13 ; 0.55$ & $1.14 ; 0.69-1.91 ; 0.60$ \\
\hline Maternal & 10.9 & Reference group & Reference group \\
\hline \multicolumn{4}{|c|}{ Carrier risk based on relationship to child with $\mathrm{CF}$} \\
\hline 1 in 2 & 31.8 & $5.56 ; 2.47-12.52 ;<0.001$ & $5.17 ; 2.38-11.24 ;<0.001$ \\
\hline 1 in 4 & 6.6 & Reference group & Reference group \\
\hline 1 in 8 & 1.0 & $-^{b}$ & $-^{b}$ \\
\hline
\end{tabular}

Abbreviations: $\mathrm{AOR}$, adjusted odds ratio; $\mathrm{Cl}$, confidence interval; $\mathrm{OR}$, odds ratio.

$N=716$.

aThere were 37 non-parent relatives for whom gender could not be confirmed by the parents in the interview and these have been excluded from this analysis of the gender variable.

bThe odds ratio could not be calculated due to the very small number of participants in this group. 
making about genetic testing. ${ }^{20}$ Tests of interaction showed that the effect of gender on test status does not depend on family background (whether or not the relative is from the maternal or paternal side; $P=0.80$ ), and hence on both sides of the family, females are more likely than males to have carrier testing. Some relatives are tested immediately after the child's diagnosis and others are still undergoing

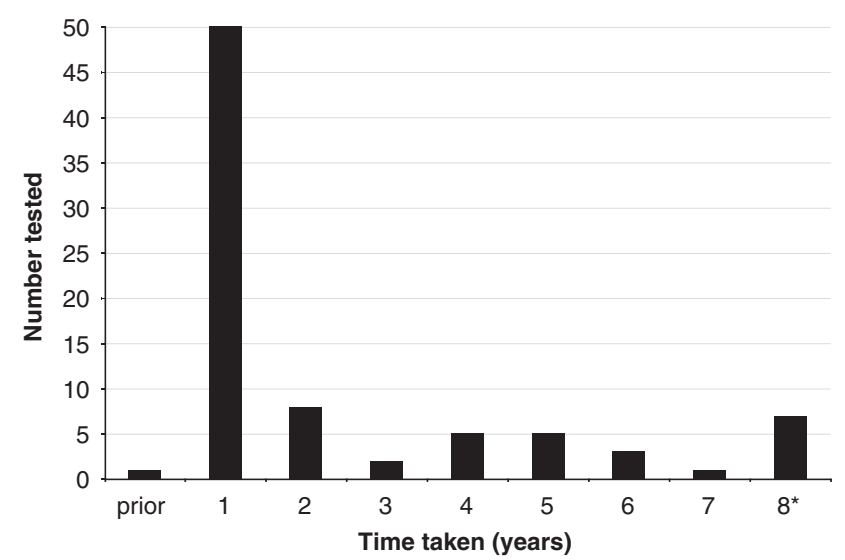

Figure 1 Time taken for non-parent relatives to have carrier testing after the diagnosis of a child with CF through newborn screening. *This column represents relatives who have been tested in the eighth year after their family member was diagnosed with CF. The database search was conducted in March 2009, and hence only tests conducted in the first quarter of 2009 have been included and this column does not represent a full year. One relative was tested 39 months before the birth of the child in this study. This was because another child in the family was born with CF 15 years previously (first cousin once removed from the child in the present study). The relative (carrier risk of 1 in 4 , based on relationship to child in this study) was tested when she was pregnant, which was before the birth of the child in this study. testing some 8 years later (Figure 1). Over half (61\%; 50/82) of nonparent relatives who are tested, however, do so within 1 year of the child's diagnosis. The age at which non-parent relatives are tested shows a bimodal distribution: one peak for those who are in their reproductive years and one peak for those who are post-reproduction (Figure 2). Stratifying the analysis for age of non-parent relatives was not possible for those who have not been tested as their dates of birth could not be collected within this study because of the Privacy Laws in the State of Victoria. Dates of birth could only be collected for relatives who have had carrier testing. In Australia, there are no recommendations that testing be restricted by the age ( $>18$ years) or life stage of relatives. $^{12}$

This study, undertaken in a clinical setting, shows that $88.5 \%$ of eligible non-parent relatives remain untested. This evaluation of clinical practice provides an estimate of uptake that is lower than that observed in research studies about carrier testing for $\mathrm{CF}$ in families in which an 'active' approach to testing is taken (ranging from 29 to $69 \%$, or described as a significant increase in numbers of tests performed per month), ${ }^{6-9}$ but a higher estimate than a 'passive' approach study $(<10 \%){ }^{10}$ The setting for our study is similar to a previous 'passive' approach in that a genetic counselling session to discuss the availability of carrier testing for relatives is offered, a pedigree is drawn and parents are provided with information to pass on to their other relatives. ${ }^{10}$ An important difference with our study is that within the clinical setting the offer of testing for relatives occurs at the same time as the child's diagnosis (during the parent education program after NBS), whereas Surh et al ${ }^{10}$ described a genetic counselling intervention undertaken as part of a research study, which was not a component of routine clinical practice, with adults with CF or parents of a child with CF. Genetic counselling at the time of diagnosis, as part of routine clinical practice in our setting, provides parents with the opportunity to discuss testing for other relatives in the context of sharing the diagnosis with their family. This may

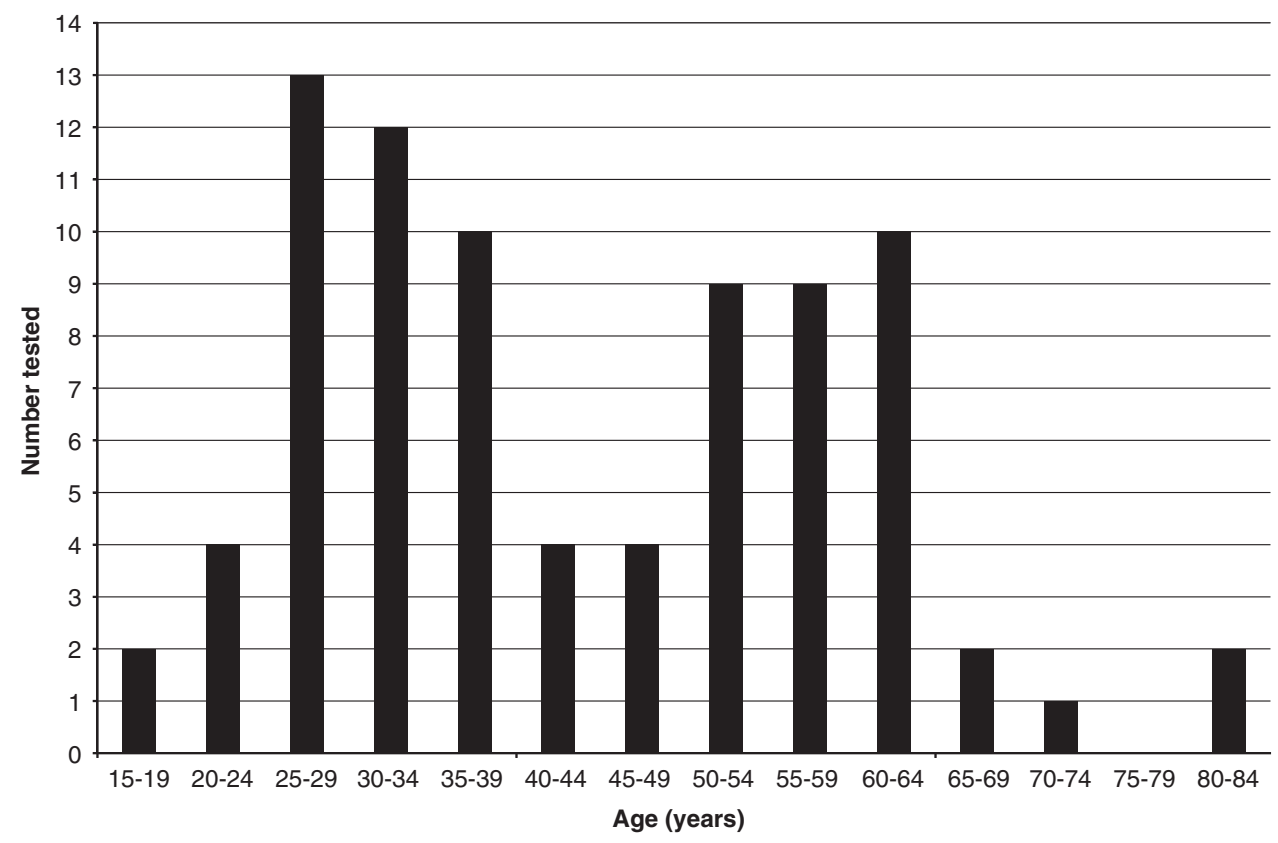

Figure 2 Age of non-parent relatives when tested. One relative was tested at age 15 , and one was tested at age 16 ; however, both were aged $\geq 18$ years at the time of the interview and hence met the study inclusion criteria. The first peak represents relatives who are in their reproductive years. Relatives who are being tested at this age may be doing so to use the information for their own reproductive planning. The second peak represents relatives who are postreproduction; these relatives may be having testing to clarify their carrier risk and to pass such information on to their own children who may be planning a pregnancy. 
explain why a higher level of uptake is observed in our study compared with the 'passive' approach study. ${ }^{10}$

Two-thirds of parents confirmed their carrier status with testing. Parents of nine children who were homozygous for the p.F508del mutation were not tested and for another three children, only one parent was tested (Table 2). The absence of testing in these parents is likely to have been based on the assumption that their genotypes could be inferred from their child's genotype; nonetheless, even in this setting, testing of both parents is of benefit to exclude the possibilities of uniparental isodisomy, ${ }^{21}$ non-paternity or sample mix-up. ${ }^{22}$ In our study we did not identify any such cases. In Australia, although nondirective genetic counselling and carrier testing is offered to parents for recurrence risk and for cascade testing, ${ }^{11}$ parents may choose to be tested or not. CF carrier testing has the potential to cause psychological harms as well as benefits; genetic counselling, to manage the effect of such harms, is therefore an important component of clinical practice. ${ }^{23}$ These harms may influence relatives' decisions about carrier testing and therefore may explain to some extent the relatively low uptake of carrier testing that we report. Further investigation of the influences on relatives' decisions about carrier testing is needed. The discovery of non-paternity is a potential harm of carrier testing parents after a child's diagnosis of CF through NBS; although this potential harm is not routinely discussed in a genetic counselling session in our setting, genetic counsellors have skills to support parents for whom this possibility is a reality. Future research could explore with parents their experience of carrier testing after their child's diagnosis of CF, and in particular seek views and attitudes regarding the value, if any, parents place on such testing. Similarly, exploring the value of carrier testing, as perceived by non-parent relatives, is necessary as this has not been clearly established.

The results from this study may underestimate the proportion of eligible non-parent relatives who have had carrier testing as it is possible that some have been tested but their result was not held in the database. Relatives who reside interstate or overseas were excluded at the beginning of the study as their records would not be held in the Victorian database. Although carrier testing for CF may be accessed through a few private laboratories in Victoria, cascade testing for relatives is provided free of charge (funded by the State Department of Human Services) and is performed by a single state-wide laboratory. Nevertheless, it is possible that some non-parent relatives who are unaware that they can have carrier testing free of charge have sought and paid for testing at a private laboratory, and as such their records would not have been included in this study. In addition, it is possible that some relatives have not been tested because their carrier status was clarified by another family member's negative result. Exploring the reasons why non-parent relatives were or were not tested was not the aim of this audit study, and therefore it remains unclear whether clarification of risk explains why some relatives have not had carrier testing.

The detection of CF carriers through NBS presents another avenue for cascade testing in the absence, however, of a diagnosis of CF in the family. ${ }^{15,16}$ Passing on the genetic information to other family members has been described as 'problematic' after a child is identified as a CF carrier through NBS. ${ }^{24}$ Policy recommendations regarding cascade testing after NBS in Victoria are to discuss cascade testing with parents of children diagnosed with CF. When a child is identified as a CF carrier after NBS, carrier testing is offered by a genetic counsellor to parents on the same day as the sweat test, and cascade testing for other adult relatives from the relevant side of the family is discussed. The main aim of this study was to audit cascade testing after NBS resulting in diagnosis of CF in a child and report the uptake of testing by non-parent relatives. As such, data were not collected on cascade testing of carriers detected through NBS. This study may have some findings that are transferable to families in which a carrier child is identified through NBS. Providing support for parents in the form of re-contact with a genetic service to discuss the implications for other relatives, sometime after the information is first known, may be beneficial to reduce the burden of communicating genetic information. The effect of the absence of a diagnosis of CF would need to be further examined, and is beyond the scope of this paper.

It is important to understand how carrier testing, after a child is diagnosed with CF through NBS, is accessed to tailor services to the needs of parents and other relatives. Positive attitudes to carrier testing, and high uptake when an 'active' approach to offering testing, as shown in the literature, suggests that non-parent relatives do want the opportunity to make a decision about carrier testing for CF for themselves, ${ }^{6-9,25,26}$ but in our study more than $88 \%$ of non-parent relatives are not tested.

This study was not designed to explain the relatively low levels of carrier testing identified. Although communication with relatives may occur for familial support after the diagnosis, discussion of genetic risk seems to occur later. ${ }^{27,28}$ It is not clear whether uptake of CF carrier testing is a true reflection of communication because relatives may choose not to be tested despite being informed of their carrier risk. Informed relatives may not access testing because: (1) they do not want to; (2) they do want to but other barriers are present, for example, they do not know how to access testing or have not pursued it yet; ${ }^{6,9,25}$ or (3) their carrier risk has been clarified by the test result of another family member. ${ }^{8}$ Further research into the decision made by non-parent relatives about carrier testing is needed to complement this study. Greater understanding of the influences of decision making for CF carrier testing may identify information needs of relatives and enhance the way clinical services such as ours are offered. We are currently undertaking a questionnaire-based survey of knowledge, attitudes and factors that influence decision making about carrier testing for non-parent relatives of a child diagnosed with $\mathrm{CF}$ through NBS.

Re-contact with parents to discuss carrier testing for other relatives could be justified. The need to assist parents with communicating to their relatives about carrier testing has been previously described. ${ }^{25,29}$ It is possible that relatives are interested but wish to decide about testing on a time frame that suits their life stage or circumstances; or that this is the pattern of familial communication in which information is disseminated to specific people at specific times. Further research is required to determine whether such re-contact is indeed justified, how such an approach would be undertaken and the most appropriate time frame for re-contact.

Previous research on cascade testing for other conditions can provide a model for how CF carrier testing could be offered to relatives of children diagnosed with CF through NBS. Newson et al $^{30}$ suggest, in their review of cascade testing for familial hypercholesterolemia, that there are two ways of how genetic information may reach relatives: (1) 'family contact' in which the existing patterns of communication are used within the family and information is disseminated by a family member, usually the proband; and (2) 'direct contact' in which relatives receive information from a clinical service; their contact details supplied to the service by the proband. They conclude that these two approaches should not compete; rather, they are complementary and could be used in succession in which the proband initially communicates to their relatives (family contact) but can draw on the clinical service to pass the information on to other relatives who may be more challenging to communicate with 
(direct contact). Similarly, studies of cascade testing for hereditary cancers describe these two approaches of making contact with relatives. $^{31,32}$ A comparison of standard clinical practice and an intervention that included greater emphasis on, and assistance with, family communication showed that with additional follow-up genetic counselling, greater proportions of relatives contacted genetic services. ${ }^{33}$ Although the studies described above can suggest how to better support families of children diagnosed with CF through NBS, an important difference is that the genetic information in the present study relates to reproductive risk rather than personal health. Further research is needed to develop an approach for offering CF carrier testing to nonparent relatives of children diagnosed with CF through NBS.

Further research is also required to determine the outcomes of carrier testing for non-parent relatives, in particular to understand how they make use of the information when making reproductive plans. Previous research examining the subsequent reproductive behaviors of parents of children with CF have documented some parents' use of prenatal diagnosis and changes in their reproductive plans, ${ }^{34-36}$ and other parents who did not have prenatal diagnosis. ${ }^{37,38}$ Parents' reproductive plans change over time; ${ }^{34,36}$ Sawyer et al $l^{34}$ recommend ensuring parents have ongoing access to genetic counselling as they make plans for future pregnancies. The same recommendation could be made for carrier couples identified in families through cascade testing.

In summary, this study has shown that approximately three nonparent relatives have CF carrier testing after a child's diagnosis with $\mathrm{CF}$ through NBS. Relatives who are most closely related to the child were more likely to be tested, and female relatives were more likely than male relatives to be tested. Nevertheless, the vast majority of nonparent relatives do not have carrier testing after a diagnosis of $\mathrm{CF}$ through NBS. These findings have implications for clinical practice for offering CF cascade carrier testing, with a suggestion to re-contact parents, after a period of time, to discuss cascade carrier testing.

\section{CONFLICT OF INTEREST}

The authors declare no conflict of interest.

\section{ACKNOWLEDGEMENTS}

We thank Ms Amanda Sherwen and the staff at the CF clinic at the Royal Children's Hospital for assistance with recruiting participants, and Dr Sharon Lewis, Murdoch Childrens Research Institute, for valuable comments on this paper. Obioha Ukoumunne is supported by the Australian NH\&MRC Capacity Building Grant 436914.

1 Southern KW, Munck A, Pollitt R et al: A survey of newborn screening for cystic fibrosis in Europe. J Cyst Fibros 2007; 6: 57-65.

2 O'Sullivan BP, Freedman SD: Cystic fibrosis. Lancet 2009; 373: 1891-1904.

3 Riordan JR, Rommens JM, Kerem B et al: Identification of the cystic fibrosis gene: cloning and characterization of complementary DNA. Science 1989; 245: 1066-1073.

4 National Institutes of Health: Consensus development conference statement on genetic testing for cystic fibrosis. Arch Intern Med 1999; 159: 1529-1539.

5 American Society of Human Genetics: Statement of the American Society of Human Genetics on cystic fibrosis carrier screening. Am J Hum Genet 1992; 51: 1443-1444.

6 Super M, Schwarz MJ, Malone G, Roberts T, Haworth A, Dermody G: Active cascade testing for carriers of cystic fibrosis gene. BMJ 1994; 308: 1462-1467

7 Sorenson JR, Cheuvront B, DeVellis B et al: Acceptance of home and clinic-based cystic fibrosis carrier education and testing by first, second, and third degree relatives of cystic fibrosis patients. Am J Med Genet 1997; 70: 121-129.

8 Turner G, Meagher W, Willis C, Colley P: Cascade testing for carrier status in cystic fibrosis in a large family. Med J Aust 1993; 159: 163-165.

9 Lafayette D, Abuelo D, Passero MA, Tantravahi U: Attitudes toward cystic fibrosis carrier and prenatal testing and utilization of carrier testing among relatives of individuals with cystic fibrosis. J Genet Couns 1999; 8: 17-36.
10 Surh LC, Cappelli M, MacDonald NE, Mettler G, Dales RE: Cystic fibrosis carrier screening in a high-risk population. Participation based on a traditional recruitment process. Arch Pediatr Adolesc Med 1994; 148: 632-637.

11 Massie J, Clements B: Diagnosis of cystic fibrosis after newborn screening: the Australasian experience-twenty years and five million babies later: a consensus statement from the Australasian Paediatric Respiratory Group. Pediatr Pulmonol 2005; 39: 440-446.

12 Human Genetics Society of Australasia Genetic Services Committee: Cystic fibrosis population screening position paper. 2009; http://www.hgsa.com.au/images/UserFiles/ Attachments/CysticFibrosisPositionPaper.pdf.

13 Massie RJ, Olsen M, Glazner J, Robertson CF, Francis I: Newborn screening for cystic fibrosis in Victoria: 10 years' experience (1989-1998). Med J Aust 2000; 172: 584-587.

14 Parsons EP, Bradley DM: Psychosocial issues in newborn screening for cystic fibrosis. Paediatr Respir Rev 2003; 4: 285-292.

15 Lagoe E, Labella S, Arnold G, Rowley PT: Cystic fibrosis newborn screening: a pilot study to maximize carrier screening. Genet Test 2005; 9: 255-260.

16 Oliver S, Dezateux C, Kavanagh J, Lempert T, Stewart R: Disclosing to parents newborn carrier status identified by routine blood spot screening. Cochrane Database Syst Rev 2004: CD003859.

17 Sawyer SM, Glazner JA: What follows newborn screening? An evaluation of a residential education program for parents of infants with newly diagnosed cystic fibrosis. Pediatrics 2004; 114: 411-416.

18 Hanley JA, Negassa A, Edwardes MD, Forrester JE: Statistical analysis of correlated data using generalized estimating equations: an orientation. Am J Epidemiol 2003; 157: 364-375.

19 Ghisletta P, Spini D: An introduction to generalized estimating equations and an application to assess selectivity effects in a longitudinal study on very old individuals. J Educ Behav Stat 2004; 29: 421-437.

20 d'Agincourt-Canning L, Baird P: Genetic testing for hereditary cancers: the impact of gender on interest, uptake and ethical considerations. Crit Rev Oncol Hematol 2006; 58: 114-123.

21 Sonnappa S, Prescott K, Adler B, Dinwiddie R, Wallis C: Cystic fibrosis and RussellSilver syndrome in a child with maternal isodisomy of chromosome 7. Pediatr Pulmonol 2005; 40: 166-168.

22 Dequeker E, Stuhrmann M, Morris MA et al: Best practice guidelines for molecular genetic diagnosis of cystic fibrosis and CFTR-related disorders-updated European recommendations. Eur J Hum Genet 2009; 17: 51-65.

23 Comeau AM, Accurso FJ, White TB et al: Guidelines for implementation of cystic fibrosis newborn screening programs: Cystic Fibrosis Foundation workshop report. Pediatrics 2007; 119: e495-e518.

24 Parsons EP, Clarke AJ, Bradley DM: Implications of carrier identification in newborn screening for cystic fibrosis. Arch Dis Child Fetal Neonatal Ed 2003; 88: F467-F471.

25 Denayer L, Evers-Kiebooms G, De Boeck K, Van den Berghe H: Reproductive decision making of aunts and uncles of a child with cystic fibrosis: genetic risk perception and attitudes toward carrier identification and prenatal diagnosis. Am J Med Genet 1992; 44: 104-111.

26 Fanos JH, Johnson JP: Barriers to carrier testing for adult cystic fibrosis sibs: the importance of not knowing. Am J Med Genet 1995; 59: 85-91.

27 Ormond KE, Mills PL, Lester LA, Ross LF: Effect of family history on disclosure patterns of cystic fibrosis carrier status. Am J Med Genet C Semin Med Genet 2003; 119: 70-77.

28 Forrest LE, Curnow L, Delatycki MB, Skene L, Aitken M: Health first, genetics second: exploring families' experiences of communicating genetic information. Eur J Hum Genet 2008.

29 Denayer L, De Boeck K, Evers-Kiebooms G, Van den Berghe H: The transfer of information about genetic transmission to brothers and sisters of parents with a CF-child. Birth Defects Orig Artic Ser 1992; 28: 149-158.

30 Newson AJ, Humphries SE: Cascade testing in familial hypercholesterolaemia: how should family members be contacted? Eur J Hum Genet 2005; 13: 401-408.

31 Cody N, Green A, McDevitt T, Lynch SA: Cascade screening in BRCA1/2 mutation carriers. Ir Med J 2008; 101: 140-142.

32 Suthers GK, Armstrong J, McCormack J, Trott D: Letting the family know: balancing ethics and effectiveness when notifying relatives about genetic testing for a familial disorder. J Med Genet 2006; 43: 665-670.

33 Forrest LE, Burke J, Bacic S, Amor DJ: Increased genetic counseling support improves communication of genetic information in families. Genet Med 2008; 10: 167-172.

34 Sawyer SM, Cerritelli B, Carter LS, Cooke M, Glazner JA, Massie J: Changing their minds with time: a comparison of hypothetical and actual reproductive behaviors in parents of children with cystic fibrosis. Pediatrics 2006; 118: e649-e656.

35 Dudding T, Wilcken B, Burgess B, Hambly J, Turner G: Reproductive decisions after neonatal screening identifies cystic fibrosis. Arch Dis Child Fetal Neonatal Ed 2000; 82: F124-F127

36 Evers-Kiebooms G, Denayer L, Van den Berghe H: A child with cystic fibrosis: II. Subsequent family planning decisions, reproduction and use of prenatal diagnosis. Clin Genet 1990; 37: 207-215.

37 Mischler EH, Wilfond BS, Fost $\mathrm{N}$ et al: Cystic fibrosis newborn screening: impact on reproductive behavior and implications for genetic counseling. Pediatrics 1998; 102: 44-52.

38 Jedlicka-Kohler I, Gotz M, Eichler I: Utilization of prenatal diagnosis for cystic fibrosis over the past seven years. Pediatrics 1994; 94: 13-16. 